\title{
Retrieving Texture Images Using Coarseness Fuzzy Partitions
}

\author{
Jesús Chamorro-Martínez ${ }^{1}$, Pedro Manuel Martínez-Jiménez ${ }^{1, \star}$, \\ and Jose Manuel Soto-Hidalgo ${ }^{2}$
}

1 Department of Computer Science and Artificial Intelligence, University of Granada \{jesus, pedromartinez\}@decsai.ugr.es

2 Department of Computer Architecture, Electronics and Electronic Technology,

University of Córdoba

jmsoto@uco.es

\begin{abstract}
In this paper, a Fuzzy Dominant Texture Descriptor is proposed for semantically describing an image. This fuzzy descriptor is defined over a set of fuzzy sets modelling the "coarseness" texture property. Concretely, fuzzy partitions on the domain of coarseness measures are proposed, where the number of linguistic labels and the parameters of the membership functions are calculated relating representative coarseness measures (our reference set) with the human perception of this texture property. Given a "texture fuzzy set", its dominance in an image is analyzed and the dominance degree is used to obtain the image texture descriptor. Fuzzy operators over these descriptors are proposed to define conditions in image retrieval queries. The proposed framework makes database systems able to answer queries using texture-based linguistic labels in natural language.
\end{abstract}

\section{Introduction}

For analyzing an image several kind of features can be used. From all of them, texture is one of the most popular and, in addition, one of the most difficult to characterize due to its imprecision. For describing texture, humans use vague textural properties like coarseness/fineness, orientation or regularity [1]2. From all of them, the coarseness/fineness is the most common one, being usual to associate the presence of fineness with the presence of texture. In this framework, a fine texture corresponds to small texture primitives (e.g. the image in figure 1(A)), whereas a coarse texture corresponds to bigger primitives (e.g. the image in figure 1(I)).

There are many measures in the literature that, given an image, capture the fineness (or coarseness) presence in the sense that the greater the value given by the measure, the greater the perception of texture 3. However, given a certain measure value, there is not an immediate way to decide whether there is a fine texture, a coarse texture or something intermediate; in other words, there is not a textural interpretation.

\footnotetext{
* This work was supported by Spanish research programme Consolider Ingenio 2010: MIPRCV (CSD2007-00018).
}

E. Hüllermeier, R. Kruse, and F. Hoffmann (Eds.): IPMU 2010, Part II, CCIS 81, pp. 542-551, 2010.

(C) Springer-Verlag Berlin Heidelberg 2010 

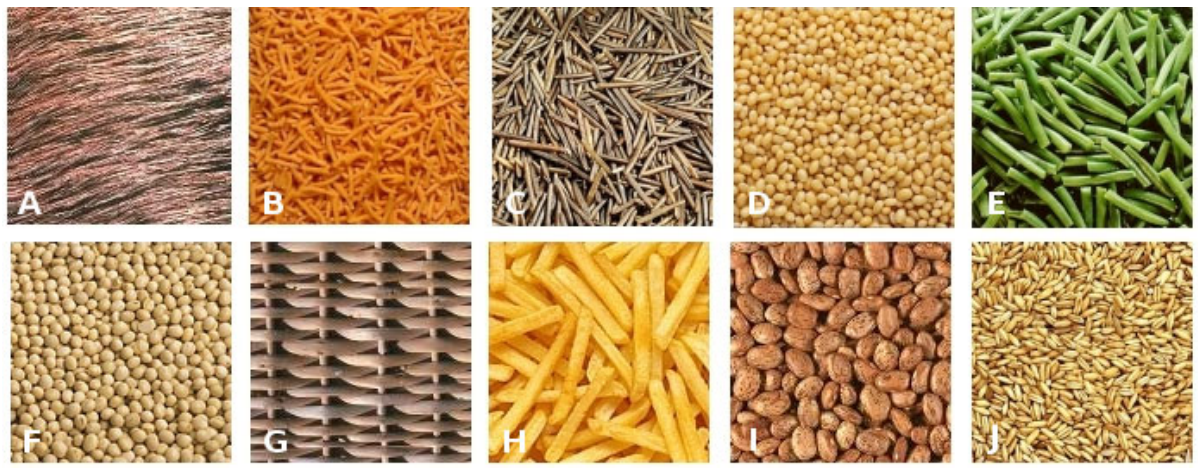

Fig. 1. Some examples of images with different degrees of fineness

To face this problem, fuzzy logic has been recently employed for representing the imprecision related to texture. In many of these approaches, fuzzy logic is usually applied just during the process, being the output a crisp result [45]. Other approaches try to model the texture and its semantic by means of fuzzy sets defined on the domain of a given texture measure. In this last framework, some proposals model the texture property by means of an unique fuzzy set [6], and other approaches define fuzzy partitions providing a set of linguistic terms 78 .

Focusing our study in the last type of approaches, two questions need to be faced for properly defining a fuzzy partition: (i) the number of linguistic labels to be used, and (ii) the parameters of the membership functions associated to each fuzzy set (and, consequently, the kernel localization). However, these questions are not treated properly in the literature. Firstly, the number of fuzzy sets are often chosen arbitrarily, without take into account the capability of each measure to discriminate between different categories. Secondly, in many of the approaches, just an uniform distribution of the fuzzy sets is performed on the domain of the measures, although it is well known that measure values corresponding to representative labels are not distributed uniformly. In addition, from our knowledge, none of the fuzzy approaches in the literature consider the relationship between the computational feature and the human perception of texture, so the labels and the membership degrees do not necessarily will match with the human assessments.

In this paper, we propose a fuzzy partition taking into account the previous questions. Firstly, in order to select the number of linguistic labels, we analyze the ability of each measure to discriminate different coarseness categories. For this purpose, data about the human perception of fineness is collected by means of a pool. This information is also used to localize the position and size of the kernel of each fuzzy set, obtaining a fuzzy partition adapted to the human perception of coarseness-fineness.

Moreover, we propose to apply the obtained fuzzy partition for texture image retrieval. The current image retrieval systems are based on features, such as 
color, texture or shape, which are automatically extracted from images. In this framework, a very important point to take into account is the imprecision in the feature descriptions, as well as the store and retrieval of that imprecise data. To deal with this vagueness, some interesting approaches introduce the use of fuzzy logic in the feature representation and in the retrieval process [9]10. These fuzzy approaches also allow to perform queries on the basis of linguistic terms, avoiding one of the drawbacks of the classical image retrieval systems, where the queries have to be defined on the basis of images or sketches similar to the one we are searching for. This way, the proposed fuzzy partition will be used to describe images in terms of their texture coarseness and the queries will be performed by using linguistic labels.

The rest of the paper is organized as follows. In section 2 we present our methodology to obtain the fuzzy partition. In section 3 a Fuzzy Dominant Texture Descriptor is proposed in order to apply the obtained fuzzy partition to texture image retrieval. Results are shown in section 4, and the main conclusions and future work are sumarized in section 5

\section{Fuzzy Partitions for Coarseness}

As it was pointed, there is not a clear perceptual interpretation of the value given by a fineness measure. To face this problem, we propose to define a fuzzy partition on the domain of a given fineness measure. For this purpose, several questions will be faced: (i) what reference set should be used for the fuzzy partition, (ii) how many fuzzy sets will compound the partition, and (iii) how to obtain the membership functions for each fuzzy set.

Concerning the reference set, we will define the partition on the domain of a given coarseness-fineness measure. From now on, we will note $\mathcal{P}=\left\{P_{1}, \ldots, P_{K}\right\}$ the set of $K$ measures analyzed in this paper, $\Pi_{k}$ the partition defined on the domain of $\mathcal{P}_{k}, N_{k}$ the number of fuzzy sets which compounds the partition $\Pi_{k}$, and $\mathcal{T}_{k}^{i}$ the i-th fuzzy set in $\Pi_{k}$. In this paper, the set $\mathcal{P}=\left\{P_{1}, \ldots, P_{K}\right\}$ is formed by the $K=17$ measures shown in the first column of table 1 It includes classical statistical measures, frequency domain approaches, fractal dimension analysis, etc. All of them are automatically computed from the texture image.

With regard to the number of fuzzy sets which compounds the partition, we will analyze the ability of each measure to distinguish between different degrees of fineness. This analysis will be based on how the human perceives the finenesscoarseness. To get information about human perception of fineness, a set of images covering different degrees of fineness will be gathered. These images will be used to collect, by means of a pool, human assessments about the perceived fineness. From now on, let $\mathcal{I}=\left\{I_{1}, \ldots, I_{N}\right\}$ be the set of $N$ images representing fineness-coarseness examples, and let $\Gamma=\left\{v^{1}, \ldots, v^{N}\right\}$ be the set of perceived fineness values associated to $\mathcal{I}$, with $v^{i}$ being the value representing the degree of fineness perceived by humans in the image $I_{i} \in \mathcal{I}$. We will use the texture image set and the way to obtain $\Gamma$ described in [11. 
Table 1. Result obtained by applying the algorithm proposed in [11]

\begin{tabular}{|l|c|c|c|c|c|c|c|}
\hline Measure & $N_{k}$ & Classes & $\bar{c}_{5} \pm \Psi_{5}$ & $\bar{c}_{4} \pm \Psi_{4}$ & $\bar{c}_{3} \pm \Psi_{3}$ & $\bar{c}_{2} \pm \Psi_{2}$ & $\bar{c}_{1} \pm \Psi_{1}$ \\
\hline Correlation [3] & 5 & $\{1,2-4,5-6,7-8,9\}$ & $0.122 \pm 0.038$ & $0.403 \pm 0.0272$ & $0.495 \pm 0.0225$ & $0.607 \pm 0.0133$ & $0.769 \pm 0.0210$ \\
ED [12] & 5 & $\{1,2,3-5,6-8,9\}$ & $0.348 \pm 0.0086$ & $0.282 \pm 0.0064$ & $0.261 \pm 0.0063$ & $0.238 \pm 0.0066$ & $0.165 \pm 0.0061$ \\
Abbadeni [13] & 4 & $\{1,2-6,7-8,9\}$ & - & $5.672 \pm 0.2738$ & $9.208 \pm 0.4247$ & $11.12 \pm 0.2916$ & $25.23 \pm 1.961$ \\
Amadasun [1] & 4 & $\{1,2-6,7-8,9\}$ & - & $4.864 \pm 0.271$ & $7.645 \pm 0.413$ & $9.815 \pm 0.230$ & $19.62 \pm 1.446$ \\
Contrast [3] & 4 & $\{1,2-5,6-8,9\}$ & - & $3312 \pm 265.5$ & $2529 \pm 295.5$ & $1863 \pm 94.84$ & $790.8 \pm 129.4$ \\
FD [14] & 4 & $\{1,2,3-8,9\}$ & - & $3.383 \pm 0.0355$ & $3.174 \pm 0.0282$ & $2.991 \pm 0.0529$ & $2.559 \pm 0.0408$ \\
Tamura [2] & 4 & $\{1,2-6,7-8,9\}$ & - & $1.540 \pm 0.0634$ & $1.864 \pm 0.0722$ & $2.125 \pm 0.0420$ & $3.045 \pm 0766$ \\
Weszka [15] & 4 & $\{1,2-6,7-8,9\}$ & - & $0.153 \pm 0.0064$ & $0.113 \pm 0.0093$ & $0.099 \pm 0.0036$ & $0.051 \pm 0.0041$ \\
DGD [16] & 3 & $\{1,2-8,9\}$ & - & - & $0.020 \pm 0.0010$ & $0.038 \pm 0.0017$ & $0.091 \pm 0.0070$ \\
FMPS [17] & 3 & $\{1,2-8,9\}$ & - & - & $0.256 \pm 0.0477$ & $0.138 \pm 0.0122$ & $0.0734 \pm 0.0217$ \\
LH [3] & 3 & $\{1,2-8,9\}$ & - & - & $0.023 \pm 0.0010$ & $0.052 \pm 0.0025$ & $0.127 \pm 0.0096$ \\
Newsam [18] & 3 & $\{1,2-6,7-9\}$ & - & - & $0.1517 \pm 0.0425$ & $0.2654 \pm 0.0466$ & $0.4173 \pm 0.0497$ \\
SNE [19] & 3 & $\{1,2-8,9\}$ & - & - & $0.879 \pm 0.0182$ & $0.775 \pm 0.0087$ & $0.570 \pm 0.0232$ \\
SRE [20] & 3 & $\{1,2-8,9\}$ & - & - & $0.995 \pm 0.00026$ & $0.987 \pm 0.00066$ & $0.966 \pm 0.0030$ \\
Entropy [3] & 2 & $\{1,2-9\}$ & - & - & - & $9.360 \pm 0.124$ & $8.656 \pm 0.301$ \\
Uniformity [3] & 2 & $\{1,2-9\}$ & - & - & - & $1.3 E^{-4} \pm 2.6 E^{-5}$ & $3.9 E_{E}^{-4} \pm 1.9_{E}^{-4}$ \\
Variance[3] & 1 & - & - & - & - & - & - \\
\hline
\end{tabular}

Using the data about human perception, and the measure values obtained for each image $I_{i} \in \mathcal{I}$, we will apply a set of multiple comparison tests in order to obtain the number of fineness degrees that each measure can discriminate (section 2.1). In addition, with the information given by the tests, we will define the fuzzy sets which will compound the partition (2.2).

\subsection{Distinguishability Analysis of the Fineness Measures}

As it was expected, some measures have better ability to represent finenesscoarseness than the others. To study the ability of each measure to discriminate different degrees of fineness-coarseness (i.e. how many classes can $P_{k}$ actually discriminate), we propose to analyze each $P_{k} \in \mathcal{P}$ by applying a set of multiple comparison tests following the algorithm shown in 11. This algorithm starts with an initial partition 1 and iteratively joins clusters until a partition in which all classes are distinguishable is achieved. In our proposal, the initial partition will be formed by the 9 classes used in our poll (where each class will contain the images assigned to it by the majority of the subjects), as $\delta$ the Euclidean distance between the centroids of the involved classes will be used, as $\phi$ a set of 5 multiple comparison tests will be considered (concretely, the tests of Scheffé, Bonferroni, Duncan, Tukey's least significant difference, and Tukey's honestly significant difference [21]), and finally the number of positive tests to accept distinguishability will be fixed to $N T=3$.

From now on, we shall note as $\Upsilon_{k}=C_{1}^{k}, C_{2}^{k}, \ldots, C_{N_{k}}^{k}$ the $N_{k}$ classes that can be discriminated by $P_{k}$. For each $C_{i}^{k}$, we will note as $\bar{c}_{i}^{k}$ the class representative value. In this paper, we propose to compute $\bar{c}_{i}^{k}$ as the mean of the measure values in the class $C_{i}^{k}$.

${ }^{1}$ Let us remark that this partition is not the "fuzzy partition". In this case, the elements are measure values and the initial clusters the ones given by the pool. 
Table 1] shows the parameters obtained by applying the proposed algorithm with the different measures considered in this paper. The second column of this table shows the $N_{k}$ classes that can discriminate each measure and the third column shows how the initial classes have been grouped. The columns from fourth to eighth show the representative values $\bar{c}_{r}^{k}$ associated to each cluster.

\subsection{The Fuzzy Partitions}

In this section we will deal with the problem of defining the membership function $\mathcal{T}_{k}^{i}(x)$ for each fuzzy set $\mathcal{T}_{k}^{i}$ compounding the partition $\Pi_{k}$. As it was explained, the number of fuzzy sets will be given by the number of categories that each measure can discriminate (shown in Table 1).

In this paper, trapezoidal functions are used for defining the membership functions. In addition, a fuzzy partition in the sense of Ruspini is proposed. Figure 2 shows some examples of the type of fuzzy partition used. To establish the localization of each kernel, the representative value $\bar{c}_{i}^{k}$ will be used (in our case, the mean). Concretely, this value will be localized at the center position of the kernel.
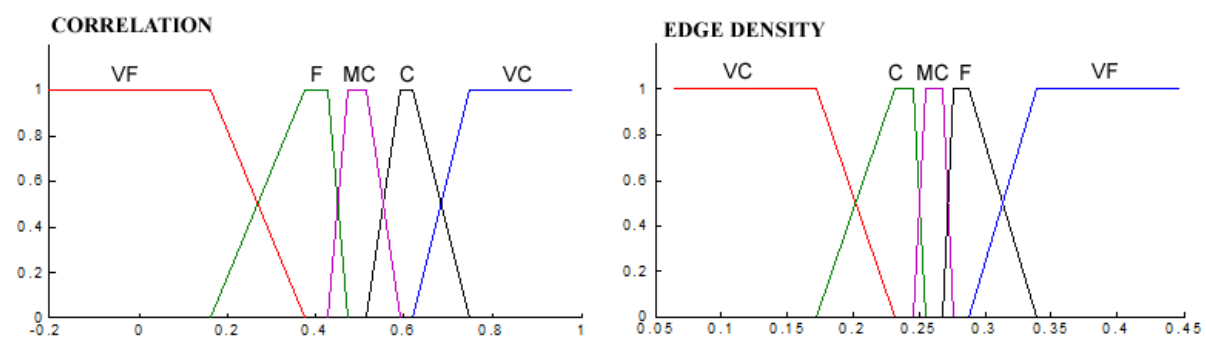

Fig. 2. Fuzzy partitions for the measures Correlation and Edge Density. The linguistic labels are $\mathrm{VC}=$ very coarse, $\mathrm{C}=$ coarse, $\mathrm{MC}=$ medium coarse, $\mathrm{F}=$ fine, $\mathrm{VF}=$ very fine.

To establish the size of the kernel, we propose a solution based on the multiple comparison tests used in section 2.1. As it is known, in these tests confidence intervals around the representative value of each class are calculated (being accomplished that these intervals do not overlap for distinguishable classes). All values in the interval are considered plausible values for the estimated mean. Based on this idea, we propose to set the kernel size as the size of the confidence interval.

The confidence interval $C I_{i}^{k}$ for the class $C_{i}^{k}$ is defined as

$$
C I_{i}^{k}=\bar{c}_{i}^{k} \pm \Psi_{i}^{k}
$$

where $\Psi_{i}^{k}=1.96 \bar{\sigma}_{i}^{k} / \sqrt{\left\|C_{i}^{k}\right\|}$, with $\bar{c}_{i}^{k}$ being the class representative value and $\bar{\sigma}_{i}^{k}$ being the estimated standard deviation for the class. Table 1 shows the values $\Psi_{i}^{k}$ for each measure and each class. 
Thus, the trapezoidal function that is used for defining the membership functions has the form

$$
\mathcal{T}_{k}^{i}(x)= \begin{cases}0 & x<a_{k}^{i} \quad \text { or } \quad x>d_{k}^{i} \\ \frac{x-a_{k}^{i}}{b_{k}^{i}-a_{k}^{i}} & a_{k}^{i} \leq x \leq b_{k}^{i} \\ 1 & b_{k}^{i} \leq x \leq c_{k}^{i} \\ \frac{d_{k}^{i}-x}{d_{k}^{i}-c_{k}^{i}} & c_{k}^{i} \leq x \leq d_{k}^{i}\end{cases}
$$

with $a_{k}^{i}=c_{k}^{i-1}, b_{k}^{i}=\bar{c}_{i}^{k}-\Psi_{i}^{k}, c_{k}^{i}=\bar{c}_{i}^{k}+\Psi_{i}^{k}$ and $d_{k}^{i}=b_{k}^{i+1}$. It should be noticed that $a_{k}^{1}=b_{k}^{1}=-\infty$ and $c_{k}^{N_{k}}=d_{k}^{N_{k}}=\infty$.

Figure 2 shows the fuzzy partitions for the measures of correlation and ED (the ones with higher capacity to discriminate fineness classes).

\section{Dominance-Based Fuzzy Texture Descriptor}

As it was pointed, we propose to apply the obtained fuzzy partition to texture image retrieval. For describing semantically an image, the dominant textures will be used. In this section, a Fuzzy Dominant Texture Descriptor is proposed (section 3.2) on the basis of the dominance degree of a given texture (section 3.1).

\subsection{Dominant Fuzzy Textures}

Intuitively, a texture is dominant to the extend it appears frequently in a given image. As it is well known in the computer vision field, the histogram is a powerful tool for measuring the frequency in which a property appears in an image. Working with fuzzy properties suggests to extend the notion of histogram to "fuzzy histogram". In this sense, a fuzzy histogram will give us information about the frequency of each fuzzy property (texture in our case). In this paper, the counting will be performed by using the scalar sigma-count (i.e., the sum of membership degrees). Thus, for any fuzzy set $T$ with membership function $T: X \rightarrow[0,1]$, the fuzzy histogram is defined a: 2

$$
h(T)=\frac{1}{N P} \sum_{x \in X} T(x)
$$

with $N P$ being the number of pixels. For texture properties, a window centered on each pixel will be used to calculate the measure value $x$.

Using the information given by the histogram, we will measure the "dominance" of a texture fuzzy set. Dominance is an imprecise concept, i.e., it is possible in general to find textures that are clearly dominant, textures that are clearly not dominant, and textures that are dominant to a certain degree, that depends on the percentage of pixels where the color/texture appears.

\footnotetext{
${ }^{2}$ In our case, this fuzzy set will correspond with the texture fuzzy set $\mathcal{T}_{k}$.
} 
It seems natural to model the idea of dominance by means of a fuzzy set over the percentages given by $h(T)$, i.e., a fuzzy subset of the real interval $[0,1]$. Hence, we define the fuzzy subset "Dominant", denoted as Dom, as follows:

$$
\operatorname{Dom}(T)= \begin{cases}0 & h(T) \leq u_{1} \\ \frac{h(T)-u_{1}}{u_{2}-u_{1}} & u_{1} \leq h(T) \leq u_{2} \\ 1 & h(T) \geq u_{2}\end{cases}
$$

where $u_{1}$ and $u_{2}$ are two parameters such that $0 \leq u_{1}<u_{2} \leq 1$, and $h(T)$ is calculated by means of Eq. 3 . We have intuitively fixed $u_{1}=0.2$ and $u_{2}=0.4$.

\subsection{Fuzzy Dominant Texture Descriptor}

On the basis of the dominance of textures, a new image descriptor is proposed for the "Texture Coarseness" property:

Definition 1. Let $\mathscr{T}$ a finite reference universe of texture fuzzy sets. We define the Fuzzy Dominant Texture Descriptor as the fuzzy set

$$
F D T D=\sum_{T \in \mathscr{T}} \operatorname{Dom}(T) / T
$$

with $\operatorname{Dom}(T)$ being the dominance degree of $T$ given by Eq. 4 .

\subsection{Fuzzy Operators}

Fuzzy operators over fuzzy descriptors are needed to define conditions in image retrieval queries. In this paper, the operators we proposed in 22 will be used.

The first one is the FInclusion (A, B) operator, which calculates the inclusion degree $A \subseteq B$, where, in our case, $A$ and $B$ are fuzzy texture descriptors. The calculus is done using a modification of the Resemblance Driven Inclusion Degree introduced in 23, which computes the inclusion degree of two fuzzy sets whose elements are imprecise.

The second one is the FEQ (A,B) operator, which calculates the resemblance degree between two fuzzy texture descriptors. The calculus is done by means of the Generalized Resemblance between Fuzzy Sets proposed in [23], which is based on the concept of double inclusion.

The described framework makes database systems able to answer queries based on the set of dominant textures within an image. Therefore, the user can define a fuzzy set of fuzzy textures (i.e, a descriptor ) which must be included in, or resemble to, the descriptor of each image in the database. Each fuzzy texture in the fuzzy set can be defined by using the linguistic labels proposed in section 2.2. which makes possible to define queries using natural language.

\section{Results}

In this section, the dominance-based fuzzy texture descriptor proposed in section 3 will be applied to texture image retrieval in order to analyze its performance. 


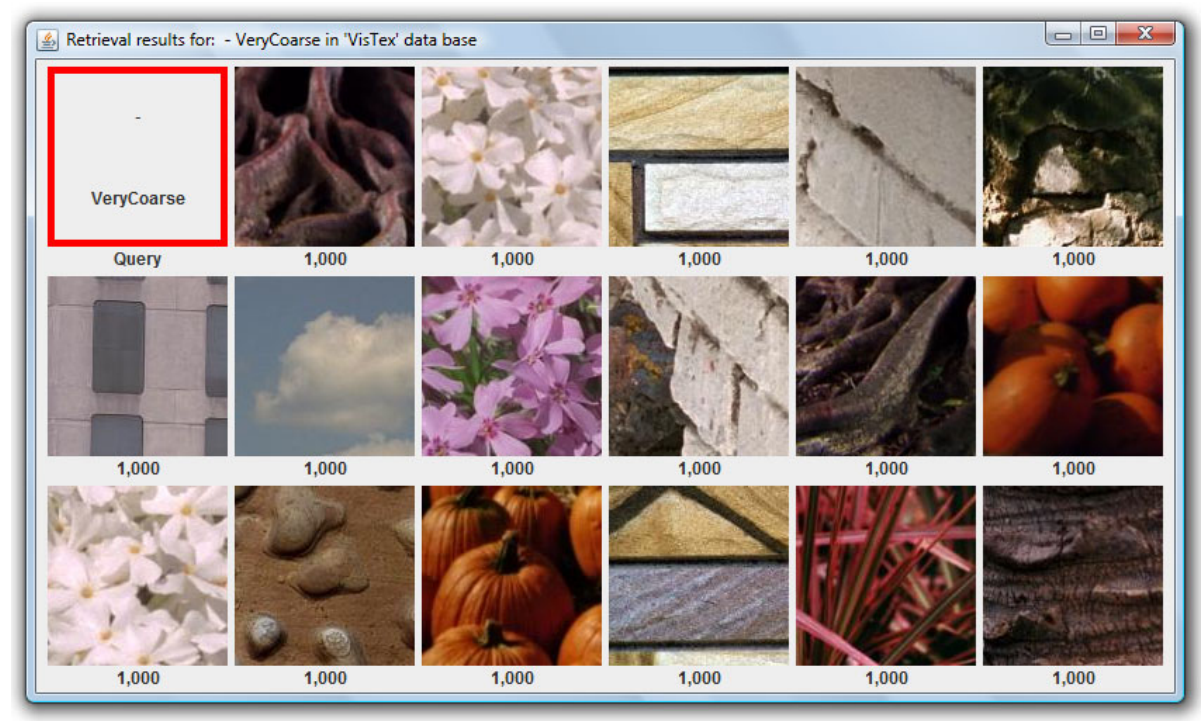

Fig. 3. Retrieval results in VisTex database using the linguistic label very Coarse as query

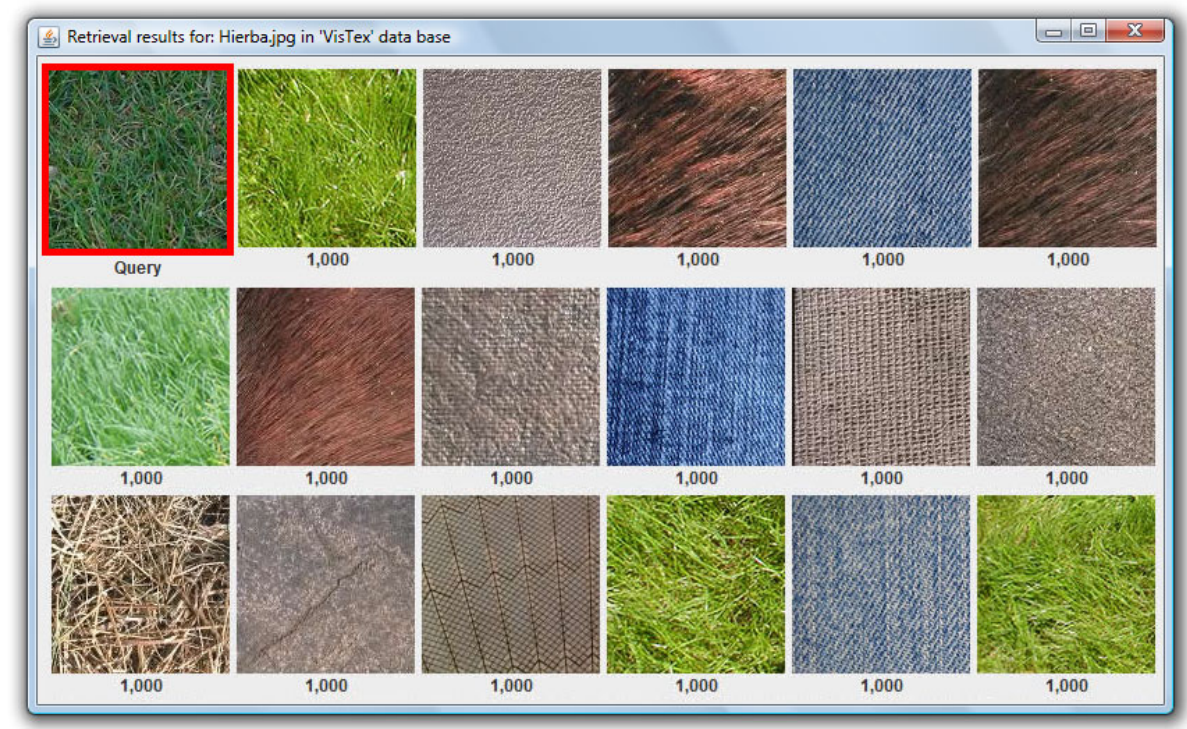

Fig. 4. Retrieval results in VisTex database using an image as query 
The fuzzy partition defined for the measure Correlation, that has the highest capacity to discriminate fineness classes, will be used.

Figure 3 shows a retrieval example using the linguistic label very coarse as query. Fuzzy dominant texture descriptor $F D T D=1$ /verycoarse has been used. The resemblance fuzzy operator described in Section 3.3 is used in this retrieval system. Figure 3 shows the retrieval results in VisTex database with resemblance degree 1 . It can be noticed that the textures of all these images are perceived as very coarse.

Figure 4 shows an example where the query has been defined by an image, i.e. we are interested in getting images with a set of dominant textures similar to the one associated with the sample image (in this case, FDTD $=1 /$ veryfine). The retrieval results with resemblance degree 1 are shown in Figure 4 and it can be noticed that the textures of all these images are perceived as very fine.

\section{Conclusions}

In this paper, a Fuzzy Dominant Texture Descriptor has been proposed for describing semantically an image. This fuzzy descriptor has been defined over a set of fuzzy sets modelling the "coarseness" texture property. Concretely, fuzzy partitions on the domain of coarseness measures have been proposed, where the number of linguistic labels and the parameters of the membership functions have been calculated relating representative coarseness measures with the human perception of this texture property. Given a "texture fuzzy set", we have proposed to analyze its dominance in an image and the dominance degree has been used to obtain the image texture descriptor. Fuzzy operators over these descriptors have been proposed to define conditions in image retrieval queries. The proposed framework has been applied to texture image retrieval in order to analyze its performance, obtaining satisfactory results.

\section{References}

1. Amadasun, M., King, R.: Textural features corresponding to textural properties. IEEE Transactions on Systems, Man and Cybernetics 19(5), 1264-1274 (1989)

2. Tamura, H., Mori, S., Yamawaki, T.: Textural features corresponding to visual perception. IEEE Transactions on Systems, Man and Cybernetics 8, 460-473 (1978)

3. Haralick, R.: Statistical and structural approaches to texture. Proceedings IEEE 67(5), 786-804 (1979)

4. Hanmandlu, M., Madasu, V.K., Vasikarla, S.: A fuzzy approach to texture segmentation. In: Proc. International Conference on Information Technology: Coding and Computing, vol. 1, pp. 636-642 (2004)

5. Barcelo, A., Montseny, E., Sobrevilla, P.: Fuzzy texture unit and fuzzy texture spectrum for texture characterization. Fuzzy Sets and Systems 158, 239-252 (2007)

6. Chamorro-Martinez, J., Galan-Perales, E., Soto-Hidalgo, J., Prados-Suarez, B.: Using fuzzy sets for coarseness representation in texture images. In: Proceedings IFSA 2007, pp. 783-792 (2007) 
7. Kulkarni, S., Verma, B.: Fuzzy logic based texture queries for cbir. In: Proc. 5th Int. Conference on Computational Intelligence and Multimedia Applications, pp. 223-228 (2003)

8. Lin, H., Chiu, C., Yang, S.: Finding textures by textual descriptions, visual examples, and relevance feedbacks. Pattern Recognition Letters 24(14), 2255-2267 (2003)

9. Hsu, C.C., Chu, W., Taira, R.: A knowledge-based approach for retrieving images by content. IEEE Transactions on Knowledge and Data Engineering 8, 522-532 (1996)

10. Sanchez, D., Chamorro-Martinez, J., Vila, M.: Modelling subjectivity in visual perception of orientation for image retrieval. Information Processing and Management 39(2) (2003) 251-266

11. Chamorro-Martinez, J., Galan-Perales, E., Sanchez, D., Soto-Hidalgo, J.: Modelling coarseness in texture images by means of fuzzy sets. In: International Conference on Knowledge-Based and Intelligent Information and Engineering Systems, vol. 2, pp. 355-362 (2006)

12. Canny, J.: A computational approach to edge detection. IEEE Transactions on Pattern Analysis and Machine Intelligence 8(6), 679-698 (1986)

13. Abbadeni, N., Ziou, N., Wang, D.: Autocovariance-based perceptual textural features corresponding to human visual perception. In: Proc. of 15th International Conference on Pattern Recognition, vol. 3, pp. 901-904 (2000)

14. Peleg, S., Naor, J., Hartley, R., Avnir, D.: Multiple resolution texture analysis and classification. IEEE Transactions on Pattern Analysis and Machine Intelligence (4), 518-523 (1984)

15. Weszka, J., Dyer, C., Rosenfeld, A.: A comparative study of texture measures for terrain classification. IEEE Transactions on Systems, Man and Cybernetics 6, 269-285 (1976)

16. Kim, S., Choi, K., Lee, D.: Texture classification using run difference matrix. In: Proc. of IEEE 1991 Ultrasonics Symposium, December 1991, vol. 2, pp. 1097-1100 (1991)

17. Yoshida, H., Casalino, D., Keserci, B., Coskun, A., Ozturk, O., Savranlar, A.: Wavelet-packet-based texture analysis for differentiation between benign and malignant liver tumours in ultrasound images. Physics in Medicine and Biology 48, 3735-3753 (2003)

18. Newsam, S., Kammath, C.: Retrieval using texture features in high resolution multi-spectral satellite imagery. In: Data Mining and Knowledge Discovery: Theory, Tools, and Technology VI, SPIE Defense and Security (April 2004)

19. Sun, C., Wee, W.: Neighboring gray level dependence matrix for texture classification. Computer Vision, Graphics and Image Processing 23, 341-352 (1983)

20. Galloway, M.: Texture analysis using gray level run lengths. Computer Graphics and Image Processing 4, 172-179 (1975)

21. Hochberg, Y., Tamhane, A.: Multiple Comparison Procedures. Wiley, Chichester (1987)

22. Chamorro-Martinez, J., Medina, J., Barranco, C., Galan-Perales, E., Soto-Hidalgo, J.: Retrieving images in fuzzy object-relational databases using dominant color descriptors. Fuzzy Sets and Systems 158(3), 312-324 (2007)

23. Marín, N., Medina, J., Pons, O., Sánchez, D., Vila, M.: Complex object comparison in a fuzzy context. Information and Software Technology 45(7), 431-444 (2003) 